\title{
Adsorption of tetracyclines on marine sediment during organic matter diagenesis
}

\author{
Ying-heng Fei and Xiao-yan Li* \\ * Environmental Engineering Research Centre, Department of Civil Engineering, The University of Hong Kong, \\ Pokfulam Road, Hong Kong, China \\ (E-mail:yhfei@hku.hk; xlia@hkucc.hku.hk)
}

\begin{abstract}
The effect of decomposition and diagenesis of sediment organic matter (SOM) on the adsorption of emerging pollutants by the sediment has been seldom addressed. In the present experimental study, artificial sediment was incubated to simulate the natural organic diagenesis process and hence investigate the influence of organic digenesis on the adsorption of tetracyclines (TC) by marine sediment. During a period of 4 months of incubation, SOM initially added into the sediment underwent biodegradation and diagenesis. The results showed an early decrease in TC adsorption by the sediment, which was likely caused by the competition between the microbial organic products and TC molecules for the adsorption sites. Afterward, TC adsorption by the sediment increased significantly, which was mainly due to the accumulation of condensed SOM. Despite of different extents of changes, the tests on three selected TCs displayed the same trend of increase in $K_{O M}$ (organic matter normalized partition coefficients). The experimental results indicate the interactions between TCs and the sediment during the dynamic process of SOM diagenesis. Moreover, the remaining SOM is shown to have an increasing affinity with the antibiotics.
\end{abstract}

Keywords: Adsorption, diagenesis, tetracyclines, sediment, sediment organic matter

\section{INTRODUCTION}

Antibiotics have been attracting more and more research attentions in recent years owing to their long-term biological side-effect in the ecosystem. Tetracyclines (TCs) are one of the most widely used antibiotic families that have drawn considerable environmental concerns (Sarmah et al. 2006). Extremely high TC concentrations that could reach $20-4000 \mathrm{mg} / \mathrm{L}$ have been reported in pharmaceutical wastewater discharges (Larsson et al. 2007). Since the conventional wastewater treatment process is ineffective for removing many antibiotics, a large portion of antibiotics in wastewater would enter natural waters via effluent and sludge disposals (Xu et al. 2007).

Adsorption is one of the most important processes related to the fate and effects of chemical pollutants in the natural environment. It has been found that TCs are strongly adsorbed by soil and sediment (Thiele-Bruhn 2003). The adsorption of antibiotics is affected by the sediment organic matter (SOM) content and composition (Thiele-Bruhn 2003; Xu and Li 2010). Under natural conditions, major fraction of the labile SOM would be mineralized biologically within a few months or years (Boudreau 1996). This SOM degradation and diagenesis might affect the adsorption behavior and capacity of SOM-rich sediment (Fei et al. 2011). In a coastal city like Hong Kong, sediment brought about by stormwater runoff, mariculture activities and sewage effluent has a high organic content ( $\mathrm{Xu}$ and $\mathrm{Li}$ 2010). There is a need to investigate the impact of organic transformation during the early SOM diagenesis on the adsorption of TCs by the sediment. However, as there are little studies on this phenomenon, the effect of SOM decay on the adsorption of chemical pollutants by marine sediment has not been well addressed. In the present laboratory study, sediment incubation experiments were conducted to simulate the dynamics of SOM 
decomposition and diagenesis in the marine sediment with a high organic content. The change of the adsorption capability and behavior of the sediment throughout the laboratory incubation was characterized.

\section{MATERIALS AND METHODS}

\section{Natural sediment}

Natural marine sediment samples were collected from the Victoria Harbour (Figure 1), Hong Kong, which are stocked below $4^{\circ} \mathrm{C}$. After shells and gravels were removed, the sediment was air-dried and homogenized gently with a mortar and pestle. It was then ground to pass through $600 \mu \mathrm{m}$ sieve for use. As influenced by the coastal discharge, the natural sediment was found to have an initial SOM content, $f_{O M}$, of around $4.9 \%$.

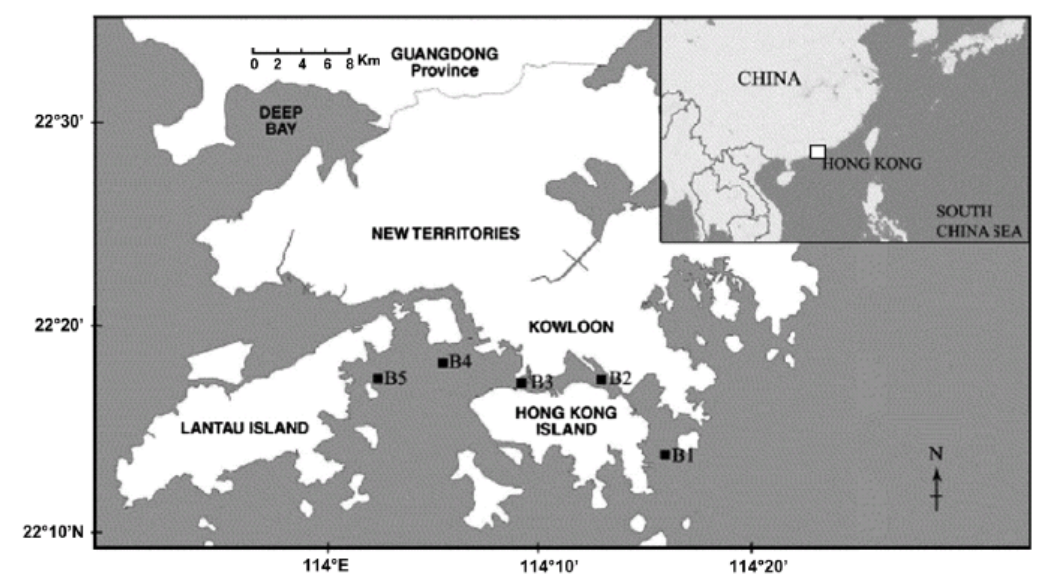

Figure 1. Map of the sediment sampling sites in Victoria Harbour, Hong Kong.

\section{Artificial sediment}

Artificial sediment with fine sand, clay and a high organic content was prepared for the experimental study. The sediment was made according to OECD guideline 218 (OECD 2004), with minor modifications. Briefly, the sediment mixture consisted of $60 \%(\mathrm{w} / \mathrm{w})$ quartz sand, $20 \%$ kaolin clay, $10 \%$ flour powder, and 10\% natural sediment. The flour powder was added as SOM, and the natural sediment was used to add SOM and microbial inoculums in the artificial sediment. The sediment was mixed thoroughly before incubation.

\section{Model tetracyclines}

As the most representative species in the family of tracyclines, tetracycline (TTC), oxytetracycline (OTC), and chlortetracycline (CTC) were selected as the model TCs for the experimental study on sediment adsorption. All of the chemicals including TCs and solvents used in the experimental study were of analytical grade or better and purchased from Sigma-Aldrich (USA). Chemical solutions were prepared just before the laboratory use.

\section{Sediment incubation}

The artificial sediment was incubated for about 4 months in marine water to simulate the early sediment diagenesis process in the estuary area with a high SOM input. The sediment was separated and placed in a number of Petri dishes that were placed on the bottom of a large water tank (Figure 2). The tank was filled with saline marine water of $10 \mathrm{psu}$ that is similar to the salinity in the estuary water of Hong Kong. The water in the tank was circulated and aerated to ensure a dissolved oxygen (DO) concentration of $5 \mathrm{mg} / \mathrm{L}$ or higher. The saline water was replaced weekly and the water 
temperature varied between $22-24^{\circ} \mathrm{C}$. Throughout the sediment incubation, one dish of the incubated sediment was retrieved from the water tank after a pre-determined time interval (about once a week in the early stage and every two weeks in the later stage) for the $f_{O M}$ determination and TC adsorption tests.

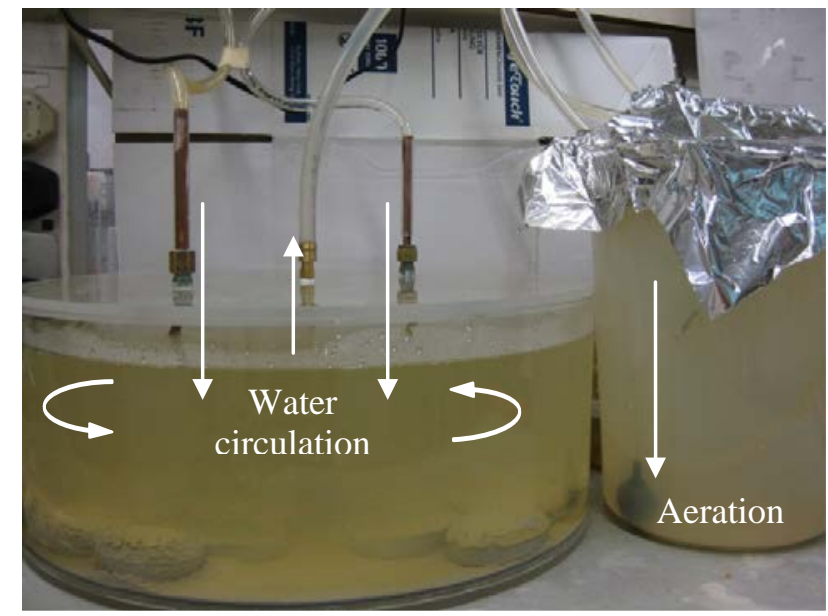

Figure 2. Incubation chamber for sediment diagenesis simulation.

\section{Batch adsorption experiments}

Batch tests on the adsorption of the model TCs by the sediment was conducted following the standard procedures which has been employed previously (Fei et al. 2011). Briefly, the sediment sample was placed in a series of conical flasks, each with $0.25 \mathrm{~g}$ dry sediment. For a model TC (TTC, OTC, and CTC), solutions of different concentrations from 2 to $20 \mathrm{mg} / \mathrm{L}$ were added into the flasks to have a volume of $30 \mathrm{~mL}$ in each flask. Sodium azide was added into the solutions at a concentration of $0.2 \%(\mathrm{w} / \mathrm{v})$ to suppress bioactivities during the adsorption tests. The flasks were sealed with parafilm and placed in a temperature-controlled shaking incubator (Polyscience, USA) at a rotating rate of 130 rpm for TC adsorption at $25^{\circ} \mathrm{C}$. All adsorption tests were conducted at pH 7.5 and a salinity of 10 psu. To investigate the adsorption kinetics, batch tests were conducted on natural sediment and non-SOM artificial sediment (sand and clay only). Samples were taken in time series and the amounts of TCs adsorbed were determined. For isotherm determination, batch tests of TC adsorption were conducted on the natural sediment, non-SOM artificial sediment and incubated sediment. The results were then used to determine the adsorption isotherm for the TC adsorption by the sediment of different incubation periods. The isotherm data were analyzed to fit with the linear partition model (Chiou et al. 1979).

\section{Chemical analysis}

The SOM contents were determined by the method similar to volatile solids in water samples. In brief, the sediment samples were dried at $108^{\circ} \mathrm{C}$ for $1 \mathrm{~h}$, and then ignited at $550^{\circ} \mathrm{C}$ for $15 \mathrm{~min}$. The weight loss after $550^{\circ} \mathrm{C}$ ignition was recorded as the $f_{O M}$.

The TC concentrations in solution were measured by an HPLC (Waters 2695) with a C18 column (5 $\mu \mathrm{m}, 2.1 \times 150 \mathrm{~mm}$ ) for separation and a photodiode array detector (Waters 2996) for quantification. For TTC and OTC, the mobile phase was a mixture of $0.1 \% \mathrm{H}_{3} \mathrm{PO}_{4}$ and methanol $(70: 30$, v/v), and for CTC, the mobile phase was changed to a mixture of $0.1 \% \mathrm{H}_{3} \mathrm{PO}_{4}$ and acetonitrile $(65: 35$, v/v). The flow rate was set at $1.0 \mathrm{ml} / \mathrm{min}$, and the sample injection volume was $10 \mu \mathrm{L}$. Under this chromatographic condition, baseline separation could be achieved within $5 \mathrm{~min}$. The area of peak at the wavelength of $275 \mathrm{~nm}$ was used for TCs quantification. The limits of detection and 
quantification for TCs were less than 0.1 and $0.3 \mathrm{mg} / \mathrm{L}$, respectively, and the relative standard deviation was below 3\%.

\section{RESULTS AND DISCUSSION}

\section{Adsorption behaviors of TTC on marine sediments}

The kinetics of TTC adsorption on the natural sediment or artificial sediment was firstly investigated. As the result indicated (Figure 3), the sediment adsorbed TTC from the aqueous phase rather quickly in the first 2-3 hr, which approached to the final equilibrium within $6 \mathrm{hr}$. Thus, all of the adsorption isotherms were then determined after $6 \mathrm{hr}$ of the batch adsorption tests.

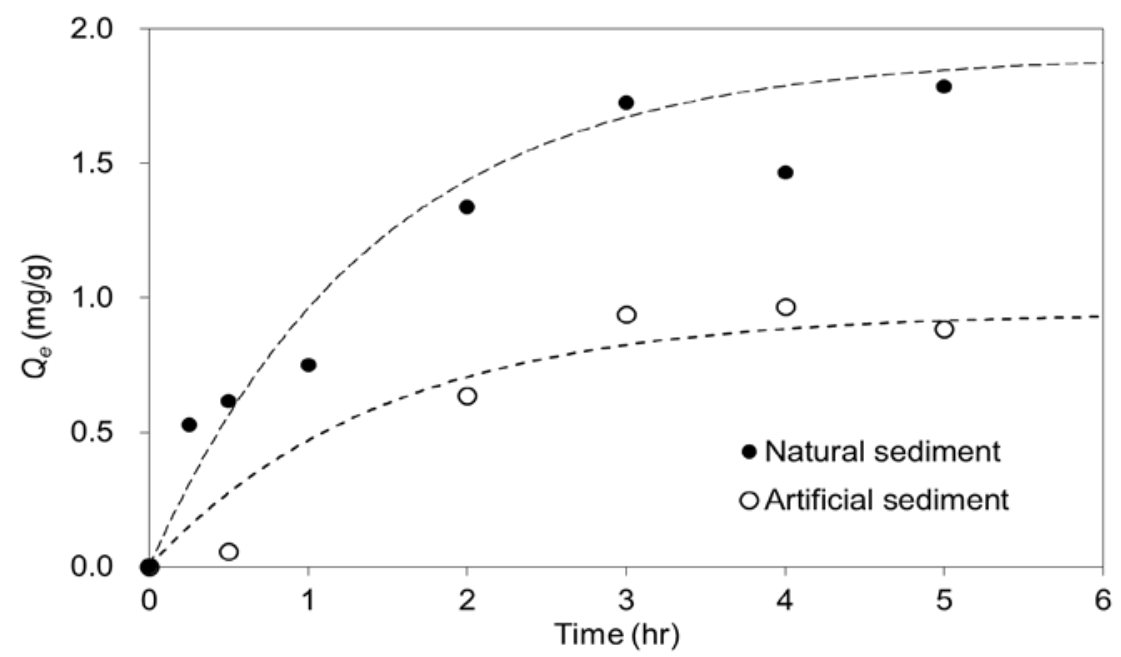

Figure 3. Adsorption kinetics of TTC on natural and artificial sediment.

As shown in Figure 4, the adsorption of TTC on the sediment followed a good linear relationship, which could be well described by the linear partition model (Eq. 1) (Chiou et al. 1979),

$$
Q_{e}=K_{d} C_{e}
$$

where $Q_{e}$ and $C_{e}$ are the equilibrated concentrations in the sediment and the aqueous phase, and $K_{d}$ is the partition coefficient indicating the adsorption capacity. As suggested by the $K_{d}$, the adsorption on natural sediment was more than 2 folds higher than the artificial sediment without SOM addition, indicating the important role of SOM in the adsorption of TTC on marine sediment.

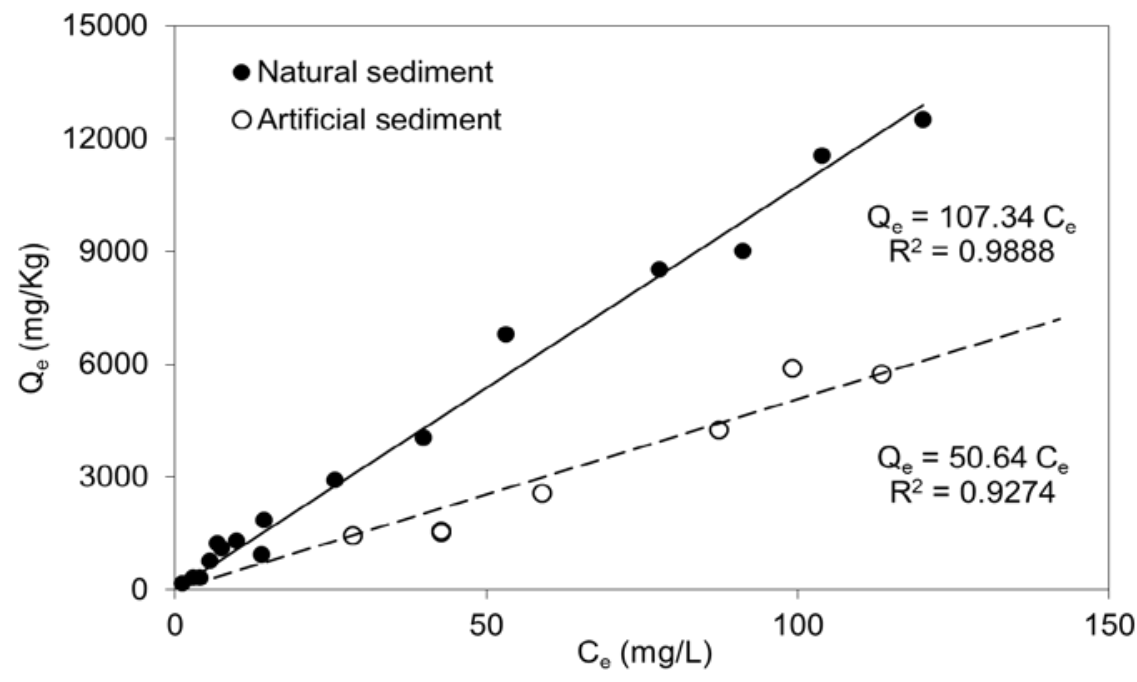

Figure 4. Adsorption isotherms of TTC on natural and artificial sediment. 


\section{SOM decomposition and biomass growth dynamics during the sediment incubation}

SOM degradation was well demonstrated by the reduction in organic content during the sediment incubation. As flour is readily biodegradable, its degradation was rapid in the 1st month of incubation (Figure 5). The organic matter fraction in the sediment decreased continuously from around $13 \%$ to less than 5\%. Afterward, the rate of SOM reduction became much slower, and the organic residue in the sediment became apparently more refractory to biodegradation.

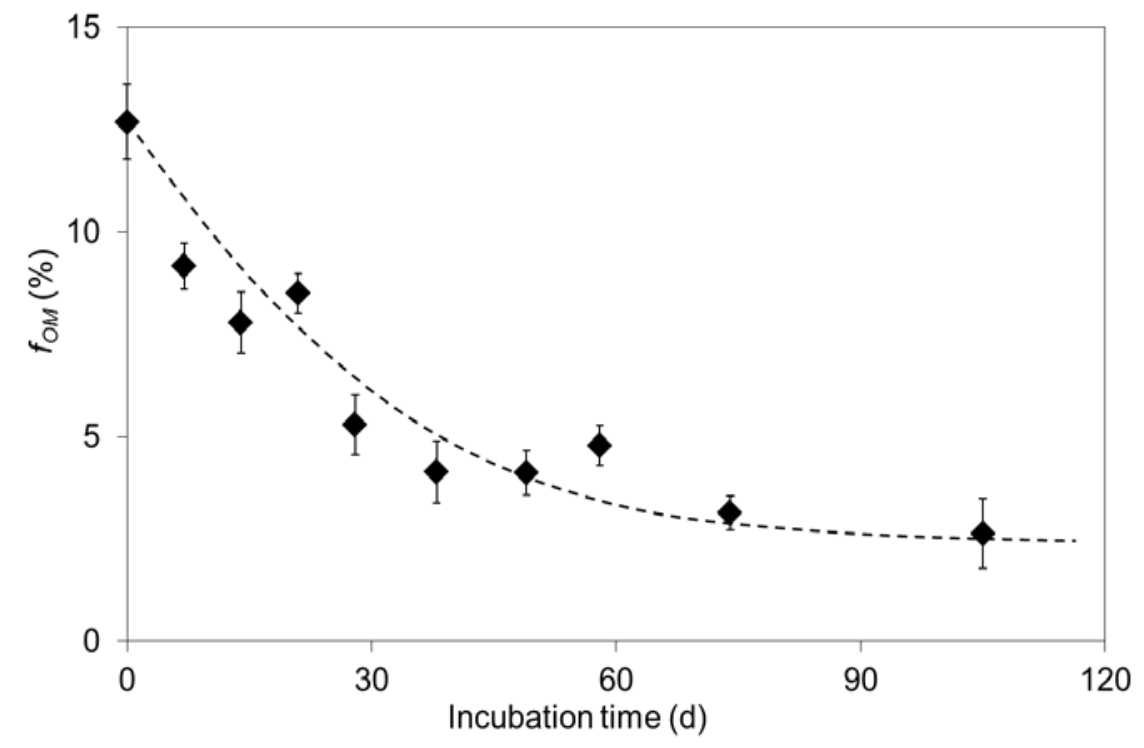

Figure 5. SOM reduction during the sediment incubation.

\section{Change of the adsorption of TCs by the sediment after incubation}

In the concentration range tested, the adsorption of TCs on the sediment can be well fitted by the linear partition model (Figure 6), and thus $K_{d}$ was obtained for TC adsorption on the sediment after various incubation periods to interpret the adsorption behaviors (Figure 7). The change in $K_{d}$ showed the impact of sediment diagenesis on the adsorption of the TCs by the sediment.

As indicated by the $K_{d}$ values, TTC adsorption by the sediment somewhat decreased in the first 2 weeks and then went upward to reach a high level at around $100 \mathrm{~L} / \mathrm{kg}$-sediment after 2 months of incubation (Figure 7a). For OTC and CTC, the $K_{d}$ values decreased considerably in the 1st month or so and then increased to reach a stable level (Figure 7b and 7c). Comparing to TTC and CTC, OTC had its $K_{d}$ decreased more significantly for the sediment after the early period of incubation, and the $K_{d}$ recovered eventually in the later phase of incubation. For TTC and CTC, the $K_{d}$ values were more than 30\% higher than their initial levels for the sediment after 3 months of incubation.

TCs can be adsorbed by both the mineral and organic components in sediment (Kulshrestha et al. 2004). The mineral fraction in sediment had little change during the incubation. However, as described above, the SOM fraction underwent significant transformation and degradation. The fresh SOM (flour) was converted to microbial biomass, which was then digested to humic-like decay products in the so-called humification process (Zech et al. 1997). The $K_{d}$ values were affected by the SOM changes during the incubation process. 

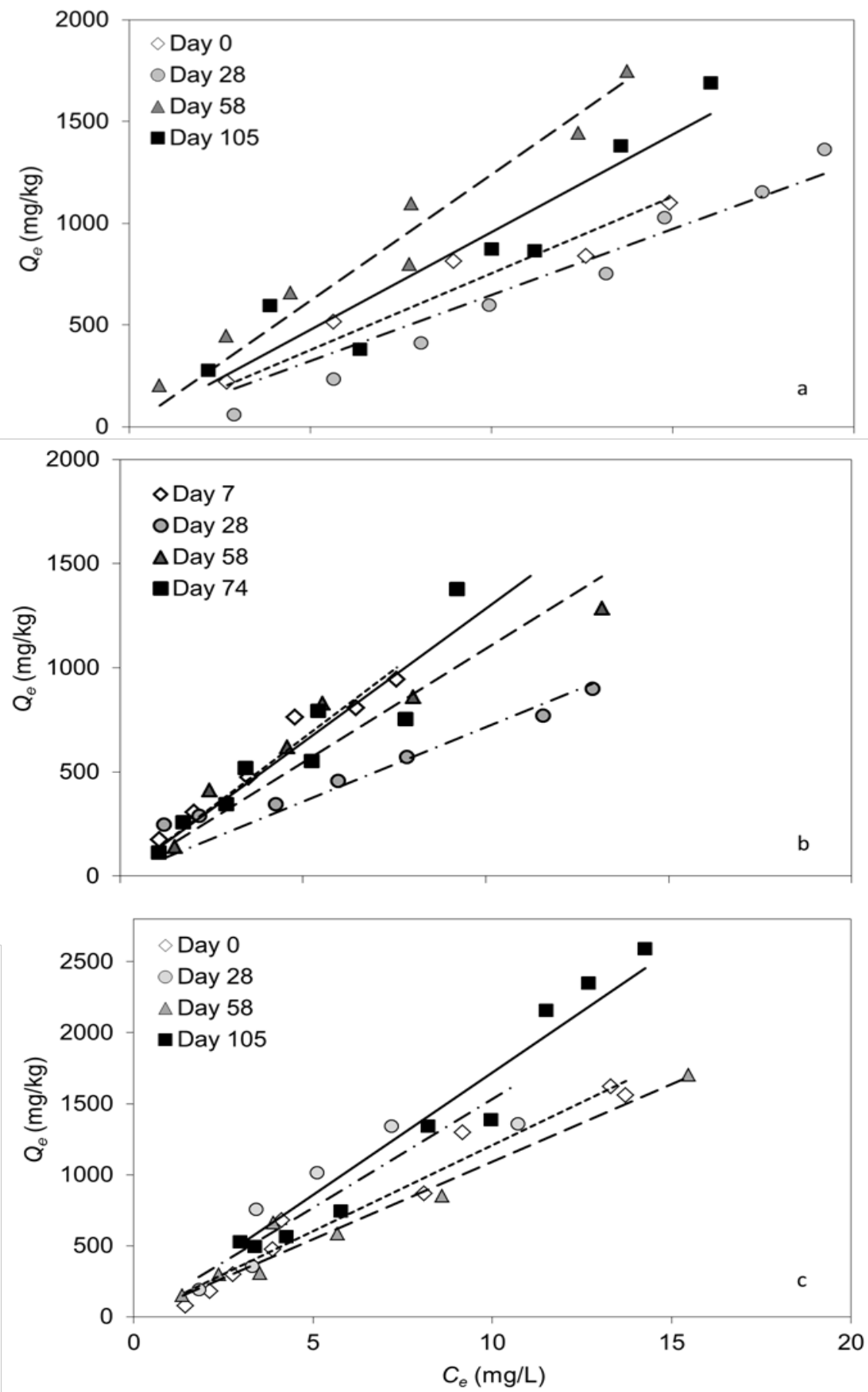

Figure 6. Adsorption isotherms of (a) TTC, (b) OTC, and (c) CTC for the sediment samples after various periods of incubation.

The initial decrease of adsorption was probably caused by the production of soluble microbial products (SMP) and extracellular polymeric substances (EPS) during the microbial growth in the sediment. SMP and EPS production would cover the surface of clay, and also interfere with the adsorption sites, and thus limited the access of TCs molecules (Pils and Laird 2007). Afterwards, SMP and EPS were degraded along with the decay of biomass and recover the adsorption by clay. Meanwhile, condensed organic matter, such as humic-like substances, was expected to be formed in the sediment (Plaza et al. 2009). Since humic matter is known to have a greater chemical adsorption capacity (Chiou et al. 1979; Pils and Laird 2007), the humification process would surely enhance the adsorption of TCs by the sediment. 


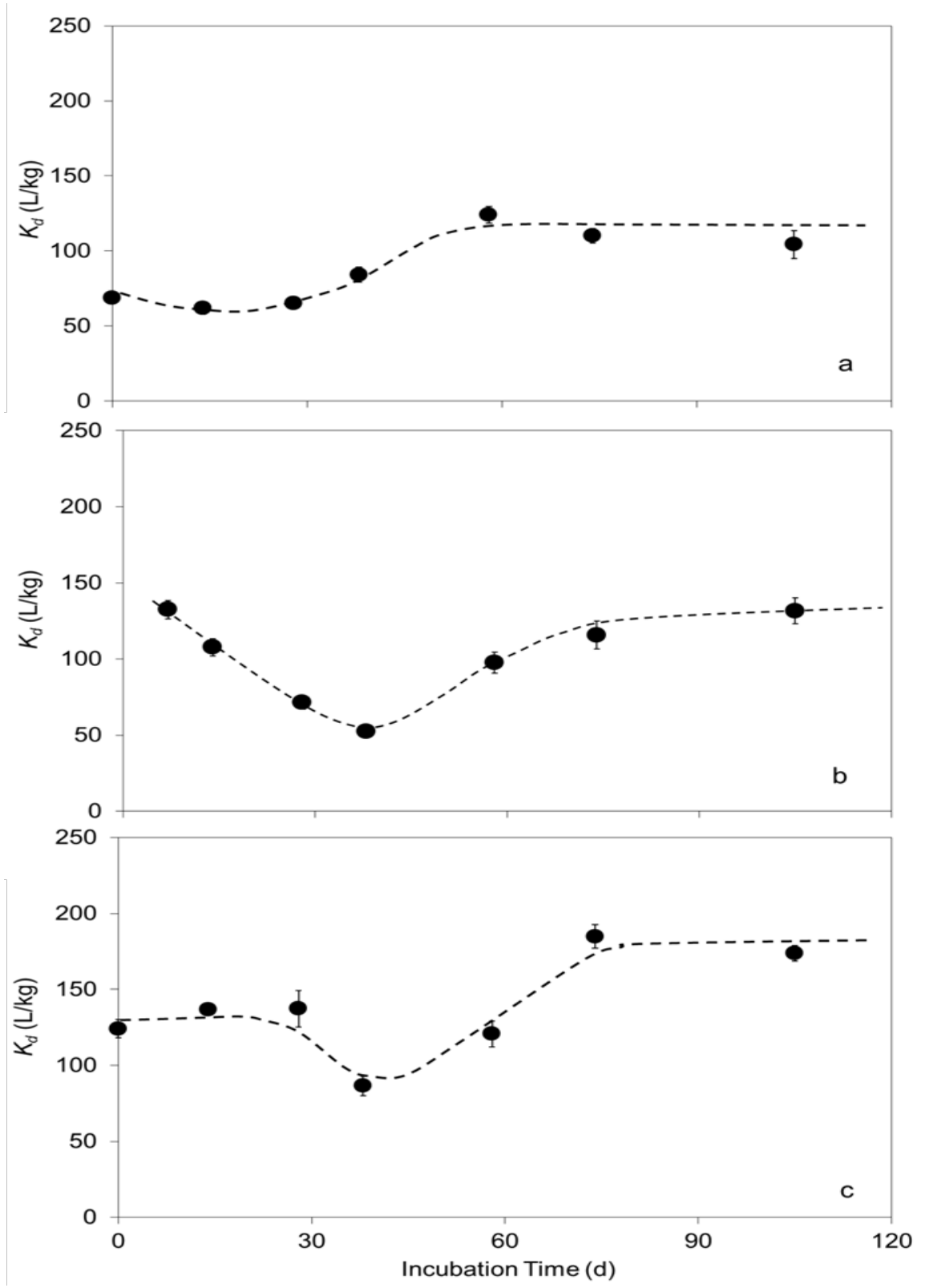

Figure 7. Change of the partition coefficients $\left(K_{d}\right)$ of (a) TTC, (b) OTC, and (c) CTC for the sediment after different periods of sediment incubation.

\section{CONCLUSIONS}

- Adsorption of selected TCs on marine sediment, either natural sediment or artificial sediment, before or after diagenesis incubation, followed linear relationship and could be well described by the linear partition model.

- Indicated by the partition coefficient, $K_{d}$, the adsorption by marine sediment was much higher than the non-SOM artificial sediment, suggesting the important role of SOM in the adsorption of TCs.

- During sediment incubation, the fresh SOM was transformed and degraded by the biomass growth and decay.

- The adsorption capacity of TCs by the sediment changed along with the diagenesis status. The adsorption was firstly decreased due to the growth of biomass and production of EPS and SMP, followed by the recovery of adsorption owing to the biomass decay and SOM humificiation. 


\section{ACKNOWLEDGEMENTS}

This research was supported by grants SEG-HKU10 and AoE/P-04/2004 from the University Grants Council (UGC) of the Hong Kong SAR Government. The technical assistance of Mr. Keith C.H. Wong is highly appreciated.

\section{REFERENCES}

Boudreau B.P. (1996) Diagenetic Models and their Implementation: Modelling Transport and Reactions in Aquatic Sediments. Springer, Berlin.

Chiou C.T., Peters L.J. and Freed V.H. (1979) Physical concept of soil-water equilibria for non-ionic organic-compounds. Sci. 206, 831-832.

Fei Y.H., Li X.D. and Li X.Y. (2011) Organic diagenesis in sediment and its impact on the adsorption of bisphenol A and nonylphenol onto marine sediment. Mar. Pollut. Bull. 63, 578-582.

Kulshrestha P., Giese R.F. and Aga D.S. (2004) Investigating the molecular interactions of oxytetracycline in clay and organic matter: Insights on factors affecting its mobility in soil. Environ. Sci. Technol. 38, 4097-4105.

Larsson D.G.J., de Pedro C. and Paxeus N. (2007) Effluent from drug manufactures contains extremely high levels of pharmaceuticals. J. Hazard. Mater. 148, 751-755.

Organisation for Economic Co-operation and Development. (2004) Test No. 218: sediment-water chironomid toxicity test using spiked sediment. In: OECD Guidelines for the Testing of Chemicals, Section 2. OECD Publishing, Paris.

Pils J.R.V. and Laird D.A. (2007) Sorption of tetracycline and chlortetracycline on K- and Ca-saturated soil clays, humic substances, and clay-humic complexes. Environ. Sci. Technol. 41, 1928-1933.

Plaza C., Xing B., Fernandez J.M., Senesi N. and Polo A. (2009) Binding of polycyclic aromatic hydrocarbons by humic acids formed during composting. Environ. Pollut. 157, 257-263.

Sarmah A.K., Meyer M.T. and Boxall A.B.A. (2006) A global perspective on the use, sales, exposure pathways, occurrence, fate and effects of veterinary antibiotics (VAs) in the environment. Chemosphere. 65, 725-759.

Thiele-Bruhn S. (2003) Pharmaceutical antibiotic compounds in soils - a review. J. Plant. Nutr. Soil. Sc. 166, 145-167.

Xu W.H., Zhang G., Li X.D., Zou S.C., Li P., Hu Z.H. and Li J. (2007) Occurrence and elimination of antibiotics at four sewage treatment plants in the Pearl River Delta (PRD), South China. Water Res. 41, 4526-4534.

Xu X.R. and Li X.Y. (2010) Sorption and desorption of antibiotic tetracycline on marine sediments. Chemosphere. 78, 430-436.

Zech W., Senesi N., Guggenberger G., Kaiser K., Lehmann J., Miano T.M., Miltner A. and Schroth G. (1997) Factors controlling humification and mineralization of soil organic matter in the tropics. Geoderma. 79, 117-161. 